\title{
DESARROLLO DE COMPETENCIAS PROFESIONALES EN EL ESPACIO EUROPEO DE EDUCACIÓN SUPERIOR (EEES). NUEVAS METODOLOGÍAS EN LOS ESTUDIOS DE COMUNICACIÓN
}

Isabel Ruiz-Mora1: Universidad de Málaga. España isabelruiz@uma.es

Silvia Olmedo-Salar: Universidad de Málaga. España silviaolmedo@uma.es

\section{RESUMEN}

Espacio Europeo de Educación Superior (EEES), nuevas metodologías docentes y competencias profesionales son algunos de los temas que centran la atención de la comunidad universitaria. Asistimos a un proceso de reconversión y adaptación, donde dejamos atrás modelos obsoletos y estáticos, aislados de la sociedad, para incluir modelos de enseñanza universitaria enfocados a vincular el mundo académico con el profesional, a través de nuevas metodologías para generar escenarios en las aulas que llaman a participar a estudiantes y docentes por igual. Entre estas metodologías encontramos el trabajo colaborativo y el juego de rol, como herramientas que potencian el trabajo en equipo y adquisición de competencias profesionales a través de la recreación de entornos reales de trabajo. Esta investigación presenta el juego de rol aplicado en el aula. Como objetivo principal pretendemos validar la inclusión de nuevas metodologías que permiten el desarrollo de competencias profesionales y potencian el trabajo colaborativo. Para ello, aplicaremos esta metodología en la materia Comunicación Política, de la licenciatura de Publicidad y Relaciones Públicas de la Universidad de Málaga. La metodología seguida para la evaluación de la actividad se ha basado en la distribución de cuestionarios entre los estudiantes que han participado en el juego del rol.

PALABRAS CLAVE: Trabajo colaborativo - Competencias profesionales - Juego de rol - Comunicación - Espacio Europeo de Educación Superior (EEES)

\footnotetext{
${ }^{1}$ Autor correspondiente

Isabel Ruiz Mora: Investigadora y docente. Facultad Ciencias de la Comunicación. Universidad de Málaga. Málaga. España

Correo: isabelruiz@uma.es
} 


\title{
PROFESSIONALS SKILLS IN THE EUROPEAN HIGHER EDUCATION AREA (EHEA). NEW METHODOLOGIES IN COMMUNICATION STUDIES.
}

\begin{abstract}
European Higher Education Area (EHEA), new teaching methods and skills are some of the themes that focus the attention of the university community. Witnessing a process of re-adaptation, where we leave behind obsolete and static models, isolated from society, to include university teaching models that linking the academic world with the professional, through new methodologies. Among these methodologies we have collaborative work and role play as tools to enhance teamwork and profesional skills through the recreation of real working environments. This research presents the role play applied in the classroom. As the main objective we intend to validate the inclusion of new methodologies for the development of professional skills and to enhance collaborative work. To do this, we will apply role play on Political Communication subject, in the Bachelor of Advertising and Public Relations (University of Malaga). The methodology for assessing the activity has been based on the distribution of questionnaires among students who have participated in the role play activity.
\end{abstract}

KEY WORDS: Collaborative work - Professional skills - Role Play Communication - European Higher Education Area (EHEA)

\section{INTRODUCCIÓN}

\subsection{EEES y nuevas metodologías}

El nuevo Espacio Europeo de Educación Superior (EEES) ha supuesto un cambio en la enseñanza universitaria, al que se han tenido que adaptar por igual docentes y estudiantes. Nos encontramos con nuevas metodologías docentes pero también, con nuevos procesos de aprendizaje, el alumnado participa de su propio aprendizaje. La clase magistral se ha quedado obsoleta, los objetivos vienen acompañados de competencias, aparece el aprendizaje autónomo y las nuevas metodologías docentes han llegado para quedarse, suponiendo para algunos un nuevo camino ppor descubrir y en el que tienen que comenzar a caminar. Son éstos y otros cambios introducidos por el conocido "Plan Bolonia", los que hacen necesaria la introducción de nuevas herramientas en el aula y los que contextualizan el presente trabajo. 
Este nuevo espacio transforma nuestro sistema educativo basado en la enseñanza, a otro basado en el aprendizaje. Además [...] se pone el énfasis en el desarrollo de competencias específicas para la profesión, así como genéricas que preparen a los futuros graduados de la universidad para su ejercicio profesional (Lara \& Rivas, 2009, p. 68)

El Real Decreto 1393/2007, de 29 de octubre es la mayor manifestación del Espacio Europeo de Educación Superior (EEES) en España. Este Real Decreto (RD) establecía la ordenación de las enseñanzas universitarias oficiales, con motivo de la adaptación de las enseñanzas universitarias al nuevo marco europeo para el año 2010. Hace un énfasis especial en el papel de las competencias, las metodologías y la evaluación:

Los planes de estudios conducentes a la obtención de un titulo deberán, por tanto, tener en el centro de sus objetivos la adquisición de competencias por parte de los estudiantes, ampliando, sin excluir, el tradicional enfoque basado en contenidos y horas lectivas. Se debe hacer énfasis en los métodos de aprendizaje de dichas competencias así como en los procedimientos para evaluar su adquisición. ${ }^{2}$

Entre los aspectos fundamentales de este RD destacan las metodologías para el aprendizaje. Como menciona Gorri Goñi (2008, p. 468), este RD se enfoca en el "cambio en las metodologías, que centra su objetivo en el proceso de aprendizaje del estudiante, en un contexto que se extiende a lo largo del ciclo vital". Por lo que, las enseñanzas deben ofrecer al estudiante las garantías suficientes para que éste prosiga con su aprendizaje autónomo una vez concluidos sus estudios superiores. Ante este nuevo contexto, los Estudios de Comunicación, al igual que el resto de estudios de la enseñanza superior española, deben adaptarse a las nuevas demandas de la sociedad actual, a los nuevos perfiles profesionales y a las propias expectativas de los estudiantes.

Las universidades viven inmersas en la sociedad que las rodea, por lo tanto se hace normal que las demandas de profesionales por parte de las empresas recalen en las instituciones educativas y éstas, las tengan en cuenta en el diseño de sus procesos de enseñanza y aprendizaje. Es por tanto necesaria, una conexión con el entorno social, laboral y económico que suponga una reconversión de la enseñanza orientada a vincular el mundo universitario con el profesional, tomando en este sentido una notable importancia la adquisición de competencias profesionales y las metodologías que facilitan las mismas.

2 Real Decreto 1393/2007, de 29 de octubre, BOE. 30 Oct. 07. Disponible en: http:// www.boe.es/boe/dias/2007/10/30/pdfs/A44037-44048.pdf.Consultado el 12/09/2011 
Según Flores, Roca y Tena (2010, p. 54) las competencias se traducen en la adquisición y aplicación de conocimientos, habilidades y actitudes que los futuros profesionales deben poseer. En palabras de estos mismos autores, la aparición de los nuevos grados se han adaptado por un lado a las exigencias educativas y tecnológicas y por otro, a las empresariales (Flores et al., 2010, p. 54). Por lo que "esta situación obliga a determinar las necesidades formativas reales para que los nuevos graduados estén perfectamente capacitados para acceder al mercado de trabajo" (Flores et al., 2010, p. 54-55). Vemos como se hace necesaria la inclusión de metodologías y herramientas docentes que faciliten la adquisición de competencias profesionales, que faciliten o ayuden a los recién titulados en su inmersión en el mundo laboral.

\subsection{Nuevas metodologías y competencias profesionales}

En los últimos años estamos asistiendo a un proceso de reconversión de las metodologías docentes orientadas a vincular el mundo profesional y el académico, promovido especialmente por el EEES. La innovación educativa está aplicando nuevas metodologías de enseñanza y aprendizaje enfocadas a propiciar nuevos entornos en las aulas, en los que los estudiantes son protagonistas de su propio aprendizaje y el docente se convierte en catalizador de las nuevas experiencias y del aprendizaje.

Entre las nuevas fórmulas destaca el juego de rol, también conocido como rol playing, caracterizado por un aprendizaje a partir de la experiencia. El juego de rol es "una herramienta de participación activa del alumno, que facilita el aprendizaje cooperativo y que, además, demuestra ser más efectiva que los métodos tradicionales para la formación en competencias" (Ortiz, Medina \& De la Calle, 2010, p. 280) y además apuntan que, "esta técnica es esencial para compatibilizar la teoría y la práctica que se requiere para la adaptación de las asignaturas al EEES, en especial las que pertenecen al ámbito de Ciencias Sociales" (Ortiz et al., 2010, p. 277).

Para Almenar, Maldonado y Hernández "consiste en desempeñar un determinado rol o personalidad concreta: cuando una persona 'hace el rol de $x^{\prime}$ significa que está realizando un papel que normalmente no hace" (Almenar, Maldonado \& Hernández, 2009, p. 2).

Es de destacar igualmente, otras posibilidades que el juego del rol brinda al potenciar habilidades fundamentales como la comunicación oral, la investigación, el análisis, el trabajo en equipo (Peterson, 1996) ${ }^{3}$. Es el trabajo en equipo o trabajo colaborativo el que favorece el aprendizaje autónomo del estudiante cuando utilizamos el juego de rol aplicado en equipos, basado en el trabajo real en grupo donde se desempeña un papel individual. Son cinco aspectos a tener en cuenta para que el trabajo en equipo funcione, tomando como referencias a Johnson y Johnson (1994) ${ }^{4}$ :

\footnotetext{
${ }^{3}$ Citado por Ortiz et al., 2010., p. 282.

${ }^{4}$ Johnson, D. W. \& Johnson, R. T. (1994): “Learning Together" en Sharan, S. (Ed.), Handbook of Cooperative Learning Methods. Greewood Press. Connecticut. Citado por Ortiz et al., 2010, p. 283
} 
a) Interdependencia positiva. Son un equipo y el trabajo de uno repercute en los

b) demás.

c) Exigibilidad personal. Cada miembro aporta al grupo su conocimiento y aprende de sus compañeros. Todo el grupo se beneficia.

d) Interacción positiva cara a cara.

e) Habilidades interpersonales y de grupo. Los estudiantes deben organizar el trabajo y tomar decisiones, manifestando sus dotes de liderazgo, conciliación,...

f) Autoanálisis del grupo. El grupo debe autoanalizarse para saber si su trabajo está siendo efectivo. Esto permite que los miembros del grupo fortalezcan sus habilidades de trabajo y fomenta el compromiso de todos con los objetivos comunes.

Por consiguiente, vemos tanto el trabajo individual como el trabajo en equipo en el juego de rol, pueden contribuir al aprendizaje individual y a la adquisición de competencias.

\section{METODOLOGÍA}

Con este trabajo en el que aplicamos el juego de rol en el aula universitaria, pretendemos determinar la validación, por parte del alumnado, de la inclusión de nuevas metodologías en el aula que permiten el desarrollo de habilidades y destrezas profesionales. Como objetivos específicos planteamos:

a) Conocer el valor que los estudiantes otorgan a las actividades, y por tanto también los aprendizajes, basados en el trabajo colaborativo

b) Determinar si el trabajo en equipo puede mejorar el aprendizaje personal

c) Averiguar qué importancia le otorgan los estudiantes a la adquisición de destrezas profesionales y por consiguiente, a las metodología que las facilitan

\subsection{Contextualización de la actividad: juego de rol en el aula}

La actividad en la que basamos nuestra investigación ha sido la aplicación del juego del rol en el aula, tuvo una planificación dos semanas y el tema era "Los emisores de Comunicación. Campañas electorales". Concretamente, se desarrolló en la asignatura de Comunicación Política de la Universidad de Málaga (curso 2010/2011), en la licenciatura de Publicidad y Relaciones Públicas.

La actividad consistía en el diseño de un programa electoral y de una campaña. En la semana 1 se hizo la presentación de la campaña y la semana 2, la entrega del programa electoral. Los grupos de trabajo estaban formados por 7/8 personas (había 69 alumnos/as matriculados) y los roles a desempeñar eran candidato, asistente del candidato. entiino encaroado del nroorama electoral v entiino encaroado de la 
La asignatura está fragmentada en dos partes, una teórica y otra práctica. La parte práctica siempre se basa en una teoría previa; por tanto, la actividad se llevó a cabo en la clase práctica ${ }^{5}$. Con este ejercicio pretendíamos acercar a los estudiantes al mundo real y profesional de la Comunicación Política realizando un ejercicio ligado a los programas electorales de los partidos políticos y los candidatos, como emisores de la comunicación. El contexto social y político en el que transcurre la actividad está mediado por las elecciones municipales del 22 de mayo del 2011, así como por la crisis económica, social y política que vive España y su entorno internacional; sin olvidar que muchos de los participantes, se enfrentaban a su primera votación electoral.

Las pautas se explicaron en clase práctica y se estructuraron de la siguiente forma:

a) Delimitación de la actividad (plazos, composición de los grupos, objetivos del trabajo, estructura)

b) Exposición de la tarea a realizar. Se ofrece información sobre lo qué se tiene que hacer, recomendaciones, recursos (clases teóricas, programas electorales de partidos políticos, legislación electoral española, manuales, bibliografía recomendada, ejemplos de debates políticos, mítines, acciones de comunicación política,...).

c) Tutorías presenciales y online.

d) Previo a este ejercicio, se han realizado varias actividades prácticas dirigidas, con ellas se pretende que los estudiantes reflexionen sobre la situación política de España y conozcan el significado de la comunicación política.

Se decide crear grupos de 7/8 personas, similar a los equipos de trabajo que participan en el desarrollo de una campaña electoral, no por el número (que es mayor) si no por la variedad de personalidades, puntos de vista, etc... que deben convivir en un entorno real de trabajo. Pretendíamos que los alumnos y alumnas conocieran las posibilidades y dificultades de trabajar con grandes grupos de trabajo.

Los roles se reparten de la siguiente forma:

a) 1 persona será el candidato/a político/a que se presente a las elecciones.

b) 1 persona será el asistente del candidato/a.

c) 6/5 personas se subdividirán en dos grupos: uno encargado del contenido del programa electoral (recomendación $4 / 5$ personas) y otro, encargado de la comunicación del mismo (recomendación 2 personas).

d) Cada grupo debe constituirse como un partido político, por tanto deben dejar

5 Agradecemos la colaboración de la profesora Paloma del Rocío López Villafranca, del departamento de Comunicación Audiovisual y Publicidad, ya que resultó de vital importancia para el desarrollo de la actividad. 
clara cuál es su ideología, símbolos, mensajes,... y actuar en base a los mismos, tanto en el programa electoral como en la presentación del mismo por el candidato/a.

De la actividad se forman 9 grupos y por tanto, obtenemos 9 partidos políticos y 9 candidatos/as. A continuación ofrecemos un calendario de la actividad expresado en fases de trabajo:

a) Fase 1. Explicamos la actividad (día 0).

b) Fase 2. Tiempo de trabajo. Contamos con dos semanas de trabajo para la elaboración del programa electoral. Se forman los grupos, se crean los partidos políticos y comienzan el juego.

c) Fase 3. El candidato y su asesor entran en acción: presentación de las propuestas. Al cabo de una semana, cada partido presenta en convocatoria pública su programa electoral.

d) Fase 4. Los frutos del trabajo se empiezan a recoger: el programa electoral. Los partidos políticos entregan su programa electoral, propuesta y planificación de la campaña electoral (semana 2).

e) Fase 5. Evaluación. Todos los participantes opinan sobre la actividad. La evaluación se realiza desde una doble vertiente: la del estudiante y la del docente ${ }^{6}$.

Llegados a este punto, nos gustaría comentar que esta actividad ha tenido un gran peso en la evaluación de la parte práctica (40\%), teniéndose en cuenta no sólo los resultados de la actividad sino también el tiempo de resolución de la actividad, la actitud del alumnado y la presentación pública de las propuestas. La evaluación se llevó a cabo mediante rúbricas.

\subsection{Evaluación de la actividad}

El diseño metodológico que hemos seguido se ha apoyado en la técnica de la encuesta, basada en preguntas abiertas y cerradas. Con este método esperamos conocer la opinión de los estudiantes sobre el juego de rol como actividad de grupo, que permite adquirir competencias vinculadas con el mundo profesional de la comunicación.

La encuesta se define como una metodología que utiliza cuestionarios estructurados como instrumentos básicos de obtención de la información y utiliza muestras que pretenden representar a la población objeto de estudio (Alvira Marín, 2004, p. 7). En palabras de Alvira Marín "en la encuesta ad hoc, el objetivo puede ser descriptivo o explicativo pero se centra en un tema de profundidad" (2004, p. 65), en este caso nos centramos en validar el juego de rol para la adquisición de competencias profesionales. 
A la vista de los objetivos planteados al inicio de la investigación, definimos la encuesta como metodología de la misma y el cuestionario como instrumento para la recolección de los datos. Las fases que hemos seguido en esta investigación han sido: planteamiento y diseño del cuestionario; selección de la muestra; recolección de datos; preparación de la información para el análisis y análisis de la información.

Se ha utilizado el cuestionario autoadministrado, ya que al tener una "población cautiva, es decir, grupos en centros escolares, centros de trabajo, etc." (Alvira Marín, 2004, p. 49), esta herramienta nos permite tener un rápido acceso y recolección de los datos, destacamos que "la gran ventaja de este método es la rapidez y el bajo coste consiguiendo siempre una alta tasa de respuestas" (Alvira Marín, 2004, p. 49).

La muestra está compuesta por los alumnos matriculados en la asignatura de Comunicación Política de la Licenciatura de Publicidad y Relaciones Públicas de la Universidad de Málaga, contamos por tanto, con una muestra cautiva. El único requisito para contestar al cuestionario era haber participado en la actividad del juego de rol durante los ejercicios prácticos de la asignatura en el curso académico 2010/2011. De los 95 estudiantes matriculados, participaron en la actividad 69 y 35 respondieron el cuestionario (un 51\% de los participantes evaluaron la actividad). La muestra queda definida por los siguientes parámetros: sexo, edad y papel desempeñado en la actividad.

a) Sexo. El 23\% de la muestra está formado por hombres y el 77\% por mujeres.

b) En cuanto a edad (en años), la distribución es la siguiente: 19 (48\%), 20 (17\%), 21 (6\%), $22(6 \%), 23(3 \%), 24(8 \%), 25$ (3\%) y más de 25 (9\%). Es de destacar que casi la mitad de los participantes (48\%) tienen 19 años y un 89\% menos de 25 .

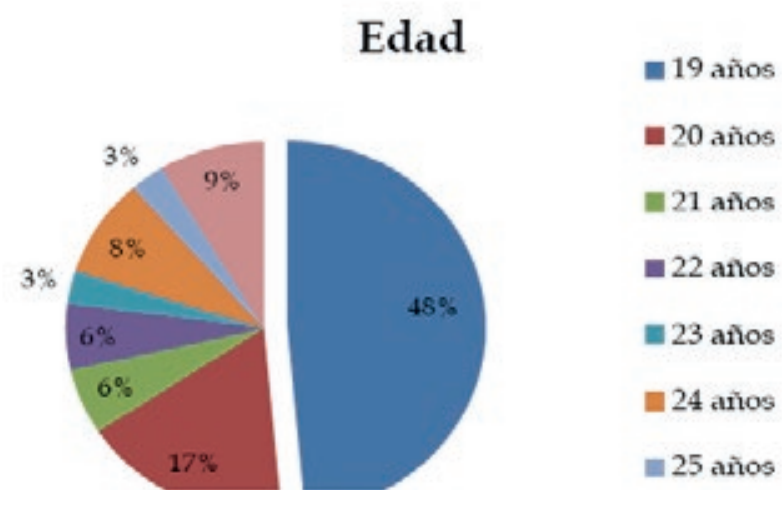

Figura 1. Distribución de la edad

c) Según la distribución de los roles: candidato/a (12\%), asistente (17\%), equipo encargado del programa electoral (40\%), equipo encargado de la campaña de comunicación $(31 \%)$. 


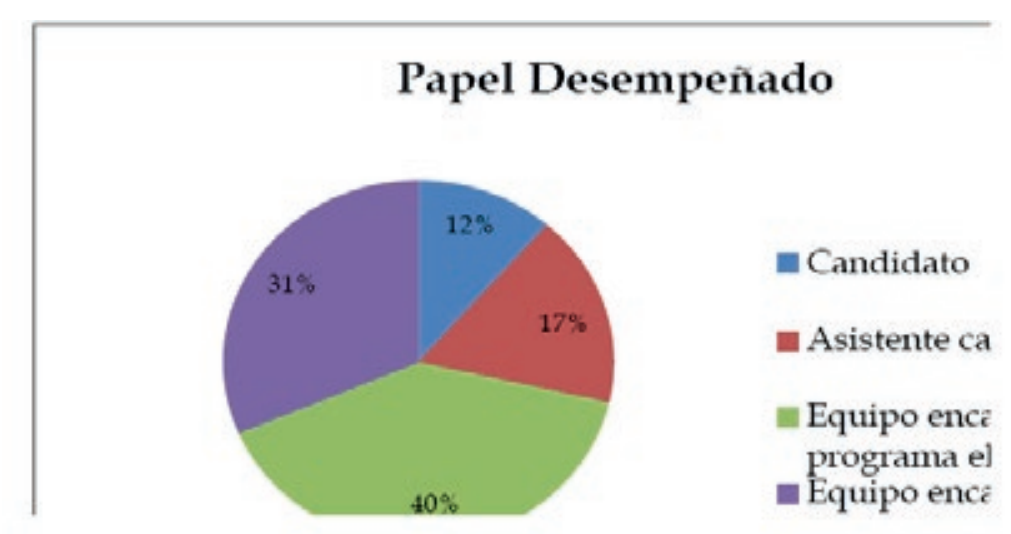

Figura 2. Distribución de los roles

\section{ANÁLISIS Y DISCUSIÓN}

A continuación presentamos los resultados de la encuesta que sirvió para que los estudiantes que había participado en la actividad, evaluaran la aplicación de la metodología del juego rol para la adquisición de competencias profesionales. Los resultados son presentados en función de las por preguntas que han compuesto la encuesta.

a) ¿Ha conseguido el desarrollo de la actividad mejorar tus conocimientos b) sobre las posibilidades profesionales de la comunicación política?

A esta pregunta el 100\% de los alumnos participantes han contestado afirmativamente, por lo que vemos que la valoración general de la actividad ha sido muy buena.

b) ¿Te han ayudado las indicaciones explicadas en clase para el desarrollo de la actividad?

En cuanto a porcentajes, el $86 \%$ ha contestado que si, un $11 \%$ que no y un $3 \%$ no han contestado.

c) ¿Consideras que el juego de rol es una herramienta válida para conocer facetas del profesional de la comunicación en el mundo de la política?

De la escala de valoración como respuesta ofrecida en esta pregunta, los resultados han sido: 
Tabla 1. Respuesta ofrecida en esta pregunta

\begin{tabular}{|l|c|}
\hline \multicolumn{1}{|c|}{ Respuesta } & $\%$ \\
\hline Totalmente en desacuerdo & $0 \%$ \\
\hline En acuerdo & $6 \%$ \\
\hline Indiferente & $6 \%$ \\
\hline De acuerdo & $54 \%$ \\
\hline Totalmente de acuerdo & $34 \%$ \\
\hline
\end{tabular}

Gran parte de los encuestados (88\%) considera que esta herramienta, el juego de rol, facilita el acercamiento al mundo profesional, en el lado opuesto, se sitúa el $12 \%$.

d) Consideras que esta actividad ha contribuido a tu aprendizaje personal...

\section{Contribución al aprendizaje}

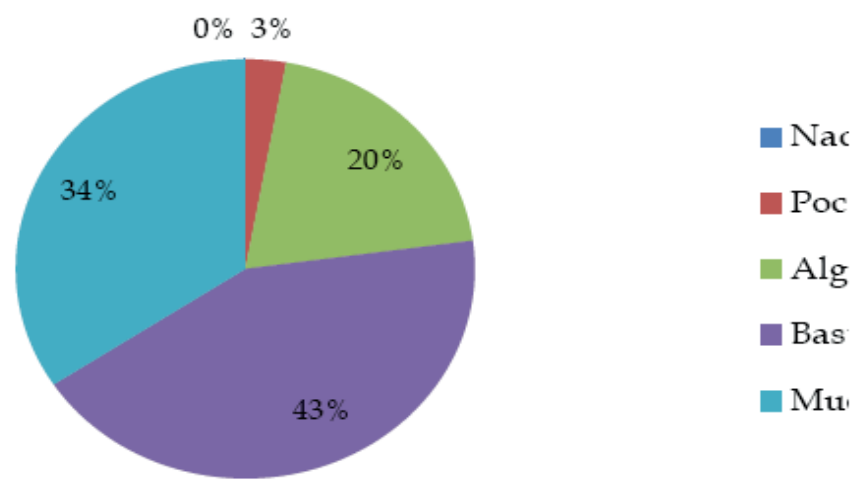

Figura 3. Distribución del aprendizaje

Como vemos un gran parte de los encuestados (77\%) consideran que el juego de rol ha contribuido a su aprendizaje personal bastante o mucho. Sólo un $20 \%$ considera que ha contribuido algo o poco $(3 \%)$ y nada $(0 \%)$.

e) ¿Cómo valorarías el trabajo en grupo como actividad básica para desarrollar todas las actividades de clase?

Con esta pregunta, pretendemos conocer cómo los estudiantes se sienten trabajando en grupo, las respuestas que hemos recogido nos transmite la postura del alumnado. Palabras como muy bien, beneficioso, útil, positivo, importante, conocimiento, acuerdo, compañerismo, distintas opiniones,... aparecen como definitorias a la hora de hablar de trabajo en equipo. Aunque también se señala problemas como reparto equitativo, mucho tiempo, fracaso, coordinador, grupo más pequeño,... que nos transmiten los problemas de esta forma de trabajar. 


\section{f) ¿Cómo valorarías los aprendizajes derivados del juego de rol??}

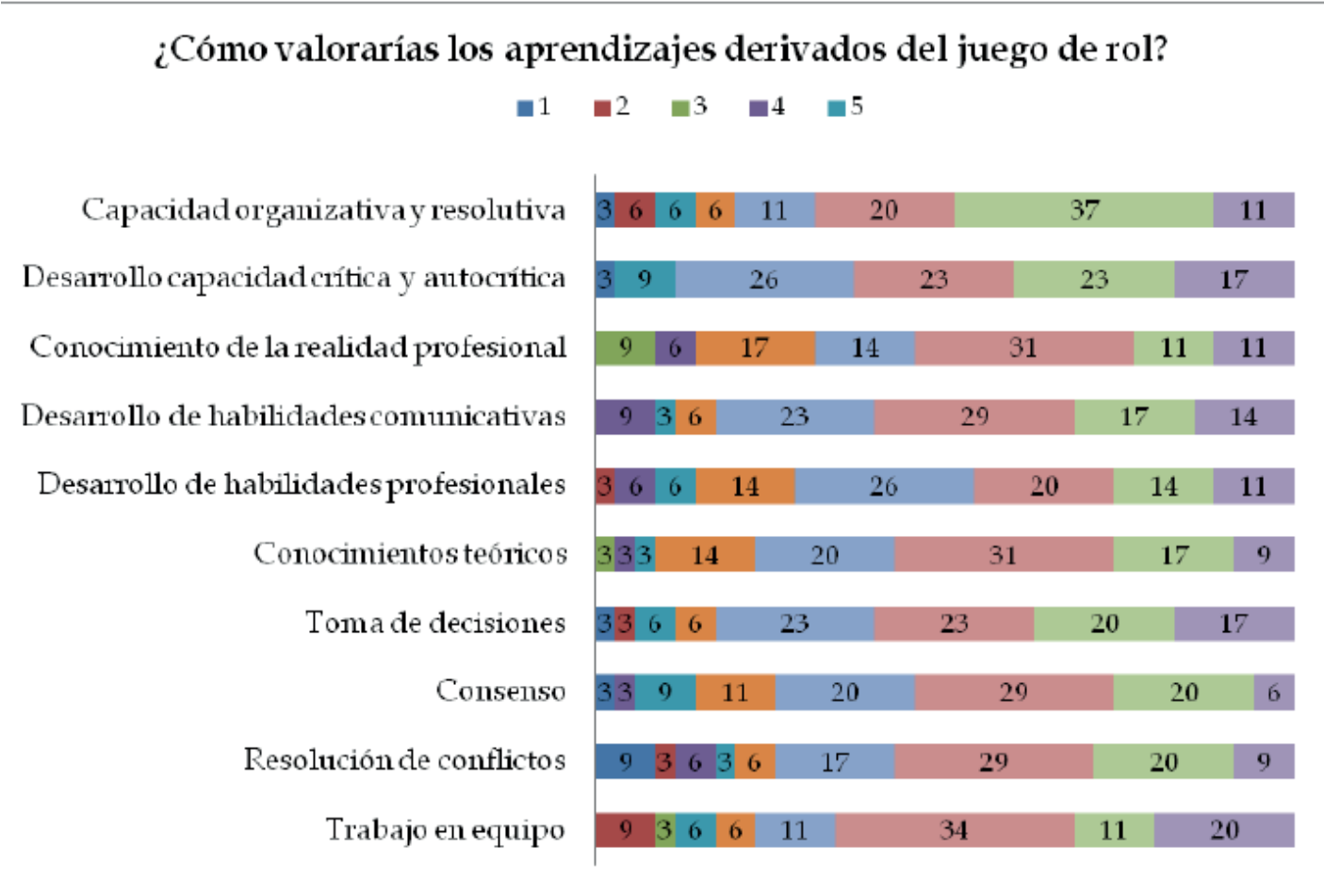

Figura 4. Distribución de los valores derivados del juego de rol

De los aprendizajes, destacamos:
a) Capacidad organizativa y resolutiva
b) Desarrollo de la capacidad crítica y de autocrítica
c) Conocimiento de la realidad profesional
d) Desarrollo de habilidades comunicativas
e) Desarrollo de habilidades profesionales
f) Conocimientos teóricos
g) Toma de decisiones
h) Consenso
i) Resolución de conflictos
j) Trabajo en equipo

g) ¿Cuál consideras que ha sido el grado de compromiso del grupo con la actividad? ¿Y el grado de compromiso individual?

Tanto el nivel de compromiso del grupo como el individual han sido considerados como bueno (37,1\% en el grupo y $42,9 \%$ en el individual) o muy bueno $(37,1 \%$ en el grupo y $42,9 \%$ en el individual) por los encuestados. Sólo un 2,9\% ha entendido que el nivel del compromiso del grupo ha sido muy malo y un $22,9 \%$ regular. Cuando 
hablamos de compromiso individual, un 14,3\% reconoce que ha sido regular, nadie estipula que su compromiso individual haya sido malo.

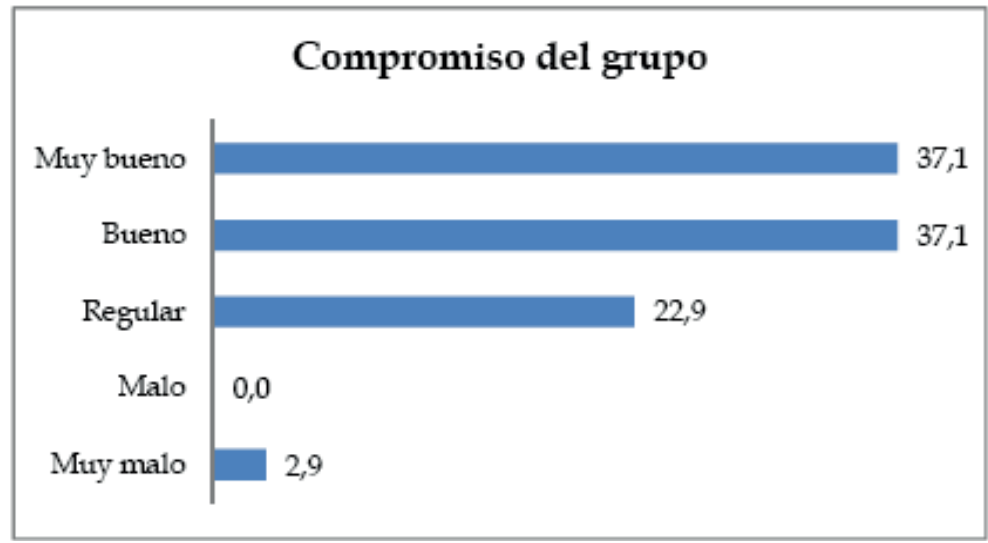

Figura 5. Compromiso del grupo

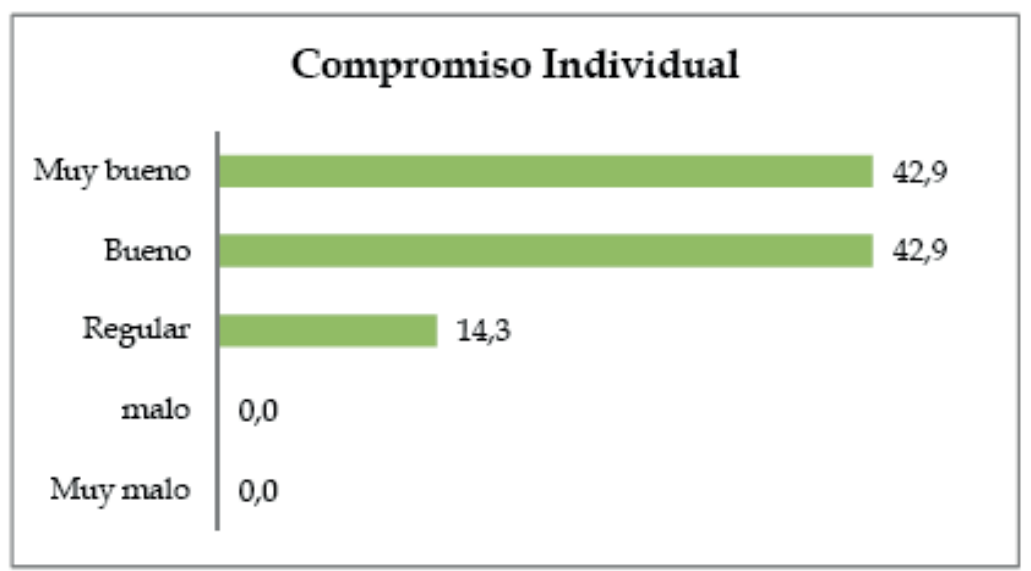

Figura 6. Compromiso individual

h) ¿Con qué otras situaciones/temas de clase se podría o te gustaría aplicar esta actividad?

Las respuestas se inclinan por actividades relacionadas con el desarrollo de alguna iniciativa ligada al mundo de la publicidad como el desarrollo de un spot del candidato político, la creación y diseño de la web de un partido político, la simulación de debates y exposiciones y otras actividades de carácter publicitario.

i) Señala una característica positiva y otra negativa de tus compañeros de grupo a la hora de planificar y realizar el juego de rol 
Tabla 2. Características del grupo

\begin{tabular}{|l|l|}
\hline Positiva & $\begin{array}{l}\text { Buenas ideas, trabajadores, interés del grupo, simpatía, unión del } \\
\text { grupo a pesar de dificultades, llegar a acuerdos, esfuerzo, } \\
\text { dedicación, alta implicación, distribución de los roles, escuchar } \\
\text { todas las opiniones, ilusión, conocernos mejor y aprender de una } \\
\text { forma práctica }\end{array}$ \\
\hline Negativa & $\begin{array}{l}\text { Problemas de organización, inexpertos, poca implicación, reparto } \\
\text { de tareas, diferencia de trabajo entre unos y otros, planificación, } \\
\text { negatividad, discusiones por horarios, decisión por el tipo de } \\
\text { partido político, diferencias ideológicas, poca implicación, } \\
\text { dificultad en el reparto de tareas }\end{array}$ \\
\hline
\end{tabular}

\section{j) Valoración general de la actividad}

\section{Valoración de la actividad}

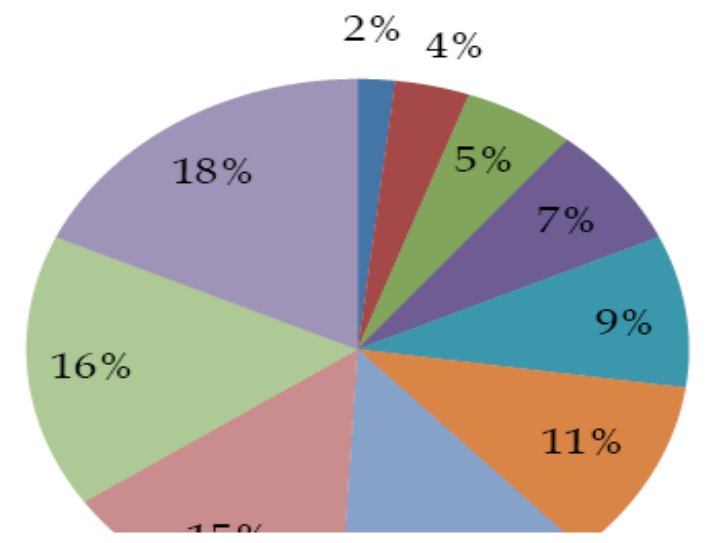

Figura 7. Valoración de la actividad

En líneas generales la actividad ha tenido muy buena acogida entre el alumnado, más de la mitad (62\%) han otorgado una calificación igual a 7 o superior, y un $18 \%$ (el mayor porcentaje) ha otorgado un 10. 


\section{k) ¿Problemas que has detectado en el transcurso de la actividad?}
a) Contenido programa electoral (qué poner)
b) Poco tiempo y mucha gente en el grupo
c) Motivación de los miembros
d) Problemas de horario
e) Excesiva importancia a la puesta en escena
f) Conflictos de grupo
g) Desconexión de las necesidades de la sociedad (falta de actualización)
h) Falta de comunicación entre los miembros del grupo
i) Diferencias culturales y políticas (alumnos con nacionalidades diferentes)
j) Pensar que no saldría bien
k) Problemas en la toma de decisiones
1) Desconocimiento de la materia
m) Problemas de ideología política

\section{CONCLUSIONES}

Llegados a este punto y tras valorar los resultados obtenidos, como conclusión principal afirmamos que el juego de rol o role playing es una técnica que facilita la adquisición de destrezas profesionales y el aprendizaje colaborativo e individual. No solamente por las afirmaciones de las investigadoras, sino por las opiniones de los participantes. A la luz de los resultados podemos observar que es una actividad muy bien valorada, nos encontramos con una buena herramienta de aprendizaje (individual y colectivo), que motiva a sus participantes, contribuye a alcanzar los objetivos de la materia y facilita la adquisición de competencias profesionales, en este caso, en el ámbito de los estudios de comunicación, focalizada en la comunicación política.

Esta actividad, por sus características y metodología, provoca entre el alumnado interés y entusiasmo; lo que favorece su desarrollo y que tenga muy buena acogida entre los participantes. Es un trabajo que no se percibe como tal, como un ejercicio más de clase, y son los estudiantes, los que proponen nuevos escenarios en los que aplicarla. El aprendizaje individual es identificado por ellos y le otorgan muy buena valoración global, podemos entonces afirmar que actividades de grupo potencian el aprendizaje individual.

Los aprendizajes que propicia el juego de rol facilitan la adquisición de competencias profesionales valoradas en el mundo profesional, destacamos la capacidad crítica y de autocrítica, el conocimiento de la realidad profesional, la capacidad organizativa y resolutiva, habilidades comunicativas, toma de decisiones o resolución de conflictos entre otras. Habilidades muy valoradas entre los egresados y valoradas por los empleadores, por qué no decirlo. 
Aprovechamos esta oportunidad que se nos brida para hacer una propuesta de mejora/recomendaciones para futuras aplicaciones del juego del rol en la enseñanza. Es conveniente la inclusión de recursos adicionales que ayuden a los participantes en el desarrollo de la actividad como son guías de trabajo en grupo, indicaciones para la solución de problemas o pautas para organización del tiempo, con ellas proporcionaríamos un mejor aprovechamiento del trabajo.

Igualmente sugerimos, que la representación del trabajo se haga pública. En esta ocasión cada grupo vivió la experiencia de manera aislada, es decir, aislados del resto de grupos; por lo que sería enriquecedor que algunas experiencias de trabajo se compartieran con el resto de participantes en el aula. Nos encontramos con una herramienta que puede servir para la autoevaluación, ya que a través del trabajo realizado por otros grupos podemos someter a evaluación el trabajo del nuestro.

Recomendamos, que la aplicación del juego de rol como ejercicio evaluable cuente con un sistema de evaluación adecuado a sus características y que éste, sea conocido por todos los participantes. Pueden resultar útiles para tal fin, el empleo de rúbricas por los evaluadores.

En materia de evaluación, apostamos porque ésta sea doble. Por un lado la evaluación del docente y por otra, la de los participantes en la actividad, tanto del desarrollo de la actividad como tal, como la del desarrollo de la actividad de otros grupos y la de ellos mismos (autoevaluación), nadie mejor que los participantes pueden valorar y premiar la consecución de la mismas, las dificultades y los aspectos mejorables. La participación de los estudiantes en la evaluación puede motivarlos a realizar un buen trabajo y ser más consecuentes con el mismo.

\section{REFERENCIAS}

Almenar Llongo, V., Maldonado Devis, M. \& Hernández Sancho, F. (2009). Una aproximación didáctica a la contratación bursátil a través de un juego de rol en google-docs. Red U. Revista de Docencia Universitaria, 7(4). Recuperado el 25 de octubre de 2011, de http:/ / redaberta.usc.es/redu/index.php/REDU/index

Alvira Martín, F. (2004). La encuesta una perspectiva general metodológica. Cuadernos metodológicos. Madrid: Centro de Investigaciones Sociológicas.

Flores Mayorga, M.Tulio, R. Correa, D. \& Tena Parera, D. (2010). La congruencia profesional de las competencias específicas del grado en Publicidad y Relaciones Públicas de las universidades públicas de Cataluña. En Competencias y perfiles profesionales en los estudios de Ciencias de la Comunicación. Madrid: Fragua. 
Gorri Goñi, A. (2008). El largo y sugestivo camino hacia el Espacio Europeo de Educación Superior. Un análisis histórico y prospectivo. Trabajo presentado al VII Congreso Estatal de Escuelas Universitarias de Trabajo Social. Recuperado el 8 de octubre de 2011, de www.trabajosocial08.com.

Lara, Sonia \& Rivas, S. (2009). Aprendizaje autorregulado y fomento de competencias en dos asignaturas de Máster a través del empleo de plantillas de evaluación, método del caso, role-playing y video digital. Educación XX1, 12: p. 67-96. Recuperado el 23 de noviembre de 2011, de

http:/ / redalyc.uaemex.mx/src/inicio/ ArtPdfRed.jsp?iCve=70611919005

Ortiz De Urbina, M., Medina Salgado, S. \& De La Calle Durán, C. (2010):

Herramientas para el aprendizaje colaborativo: una aplicación práctica del juego de rol. Teoría de la Educación: Educación y Cultura en la Sociedad de la Información, 11(3): 277-300. Recuperado el 26 de noviembre de 2011, de http:/ / campus.usal.es/ revistas_trabajo/index.php/revistatesi/article/view/7463/ 7479

Real Decreto 1393/2007. (2007). Boletín Oficial del Estado 30/10/2007. Recuperado el 20 de septiembre de 2011, de

http:/ / www.boe.es/boe/dias/2007/10/30/pdfs/A44037-44048.pdf (Consultado el).

\section{Isabel Ruiz Mora}

Es relaciones públicas, investigadora y docente en el Departamento de Comunicación Audiovisual y Publicidad de la Universidad de Málaga. Desarrolla su actividad investigadora en el ámbito de la comunicación organizacional, más concretamente en la relación que guarda las Relaciones Públicas con la Responsabilidad Social Actualmente es coeditora de la publicación, Revista Internacional de Relaciones. Pública y miembro de diferentes asociaciones como Asociación de Investigación en Relaciones Públicas (AIRRPP), Instituto de Investigación en Relaciones Públicas (IIRP), Asociación de Investigación en Comunicación (AEIC) y de European Communication Research and Education Association (ECREA).

\section{Silvia Olmedo Salar}

Periodista, docente e investigadora en el Departamento de Periodismo de la Facultad de Ciencias de la Comunicación de la Universidad de Málaga. Es miembro de la Asociación de Investigación en Comunicación (AEIC), del Instituto de Investigación en Relaciones Públicas (IIRP) y de la European Communication Research and Education Association (ECREA). Como periodista ha desarrolla la mayor parte de su trabajo profesional en la Agencia EFE y ha colaborado en radio, prensa y televisión. 